\title{
Spontaneous pneumoretroperitoneum in a renal transplant recipient
}

\author{
M.J. Gleeson and J.P. McMullin \\ Department of General Surgery/Renal Transplantation, IBN Al Bitar Hospital, Baghdad, Iraq.
}

Summary: A case of a spontaneous pneumoretroperitoneum in a 16 year old renal transplant recipient is reported. This fatal complication of immunosuppression has not been reported previously.

\section{Introduction}

The use of immunosuppression in organ transplantation is necessary to prevent rejection but may subject the patients to possible overwhelming sepsis. In many centres cyclosporin is now the form of immunosuppression used most commonly, and the use of prednisolone has been implicated as a risk factor of life-threatening infections. ${ }^{1}$

We report the case of a fatal infectious complication in a renal transplant recipient receiving azathioprine and prednisolone immunosuppression.

\section{Case report}

A 16 year old boy presented with a 2-day history of generalized abdominal pain and fever. Nine months previously he was the recipient of a renal transplant from a living related donor. Prior to this admission his serum creatinine level was stable at $233 \mu \mathrm{mol} / 1$ and his immunosuppression was achieved with azathioprine $75 \mathrm{mg}$ and prednisolone $10 \mathrm{mg} /$ day.

On admission he was apyrexial, dehydrated and had generalized abdominal tenderness with guarding. His renal transplant was non-tender. His serum creatinine level was $253 \mu \mathrm{mol} / 1$ and his leucocyte count was $7.9 \times 10^{9} / 1$. X-rays showed the presence of a pneumoretroperitoneum (Figure 1). The patient was commenced on intravenous gentamicin, ampicillin and metronidazole, and a laparotomy was performed. The findings were free, thin pus in the peritoneal cavity, bilateral discoloured paracolic gutters and release of free gas and fluid when these were opened. There was no bowel perforation. The peritoneal cavity and retroperitoneum were irri-

Correspondence: M. Gleeson, F.R.C.S.I., Department of Urology, Baylor College of Medicine, Texas Medical Center, Houston, Texas 77030, USA.

Accepted: 30 June 1988

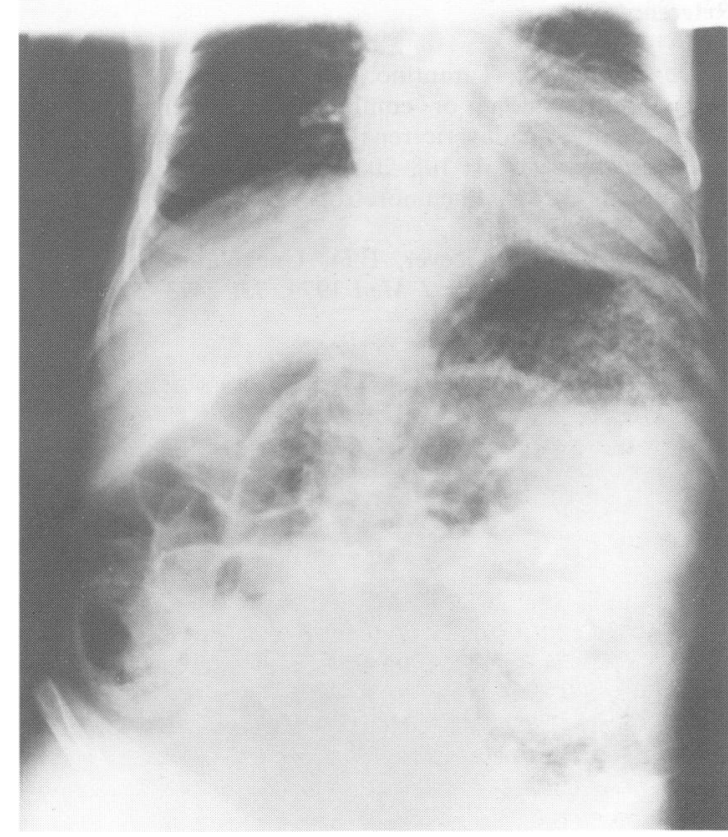

Figure 1 Erect abdominal X-ray showing pneumoretroperitoneum.

gated with saline and drained by suction drains. Blood cultures taken on admission, and swabs from the peritoneum and retroperitoneum grew Escherichia coli. A midstream urine sample taken on admission was sterile.

Postoperatively the patient developed septicaemic shock, renal failure and disseminated intravascular coagulation and died 3 days later. An autopsy was not performed.

(C) The Fellowship of Postgraduate Medicine, 1988 


\section{Discussion}

Pneumoretroperitoneum is a rare condition which may occur as a result of perforation of a hollow viscus such as the duodenum or rectum, diagnostic pneumatography, pneumatosis cystoides intestinalis, mechanical ventilation or infection. ${ }^{2}$

Although immunosuppression may subject patients to potentially fatal episodes of infection, its relationship to retroperitoneal infections has not been highlighted in the past. Retroperitoneal infection by a gas-producing organism is most likely to be renal in origin. ${ }^{3}$ Such cases of emphysematous pyelonephritis usually occur in female diabetics over 30 years of age. In $50 \%$ of cases $E$. coli is implicated as the causative organism. ${ }^{4}$ To date

\section{References}

1. De Vechhi, A., Tarantino, A., Montagnino, G. et al. Ciclosporin alone or combined with steroid in the treatment of cadaveric renal transplant recipients. Clin Transplant 1987, 1: 198-202.

2. Hillman, K.M. Pneumoretroperitoneum. Anaesthesia 1983, 38: 136-139.

3. Rosen, R.A. \& Geever, E.D. Unexplained retroperitoneal air. NY State J Med 1973, 73: 2473-2476. there has been one reported case of emphysematous pyelonephritis in a transplanted kidney. This patient was a diabetic receiving azathioprine and prednisolone as in our case. ${ }^{5}$ Although infection $\stackrel{\overrightarrow{0}}{\overrightarrow{0}}$ alone may cause a pneumoretroperitoneum, ato laparotomy was indicated in our patient to lavage 등 the areas and to outrule any possible bowel perforation. Our patient's sterile urine culture leaves the $\mathbb{\nabla}$ source of his $E$. coli infection unresolved. We feel that immunosuppression may be implicated in both ${ }^{\text {c }}$ the development of his spontaneous pneumoretro- $\vec{D}$ peritoneum and his fatal outcome despite appropriate antibiotic treatment.

This is the first reported case of a spontaneous pneumoretroperitoneum in a renal transplant recipient.

4. Costas, S. Renal and perirenal emphysema. Br $J$ Urol 1972, 44: 311-319.

5. O'Donnell, D., Rumrak, M. \& Anderson, J. Emphysematous pyelonephritis in a transplanted kidney. Clin Nephrol 1986, 25: 52-53. 